\title{
Synthesis of Some New 2-Methyl -1,4-benzothiazine-3(1H - one Derivatives as Potential Vasodilators
}

\author{
M. B. DESHMUKH*, A. R. MULIK \\ and SAVITA DHONGADE-DESAI. \\ Department Of Chemistry, \\ Shivaji University, Kolhapur 416004, India
}

Received 29 April 2004; Accepted 9 June 2004

\begin{abstract}
The increase in the internal diameter of a blood vessel that results from relaxation of smooth muscle within the wall of the vessel is vasodilation. This causes an increase in blood flow and a decrease in systemic vascular resistance. Some substances produced by tissue deprived of fresh blood seem to be responsible for the dilation. Many products of metabolism bring about the action, $\mathrm{CO}_{2}$ and acids are among them. Dilation of vessels is necessary to restore local environment of tissues and normal metabolism. It may prove to be potential in the treatment of different cardiac disorders like atherosclerosis, where, the blood vessels are narrowed due to deposition of plaque of substances like cholesterol etc. 1,4-benzothiazines have been reported to possess wide range of pharmacological and biological activities. Here, we report the synthesis and biological activity of some new arylidenehydrazino-(1H)-1,4benzothiazines. The synthesized compounds were subjected to a prediction of biological activities. A software application (PASS) was used for this purpose. The relationship between structure and different biological activities was studied and it was found that the arylidenehydrazino-(1H)-1,4-benzothiazines are expected to be potential vasodilators.
\end{abstract}

Key words :Potential Vasodialtor

\section{Introduction}

2-Methyl-1,4-benzothiazine-3(1H)-one $(1)$ was obtained by refluxing 2-aminothiophenol and methyl 2chloropropionate in $33 \% \mathrm{NaOH}$. The compound 1 was reacted with hydrazine hydrate in methanol to get 3-hydrazino-2-methyl-(1H)-1,4-benzothiazine (2), which when reacted with various substituted aldehydes furnished arylidenehydrazino-(1H)-1,4-benzothiazine (3). [Scheme ] 


\section{SOHEME}

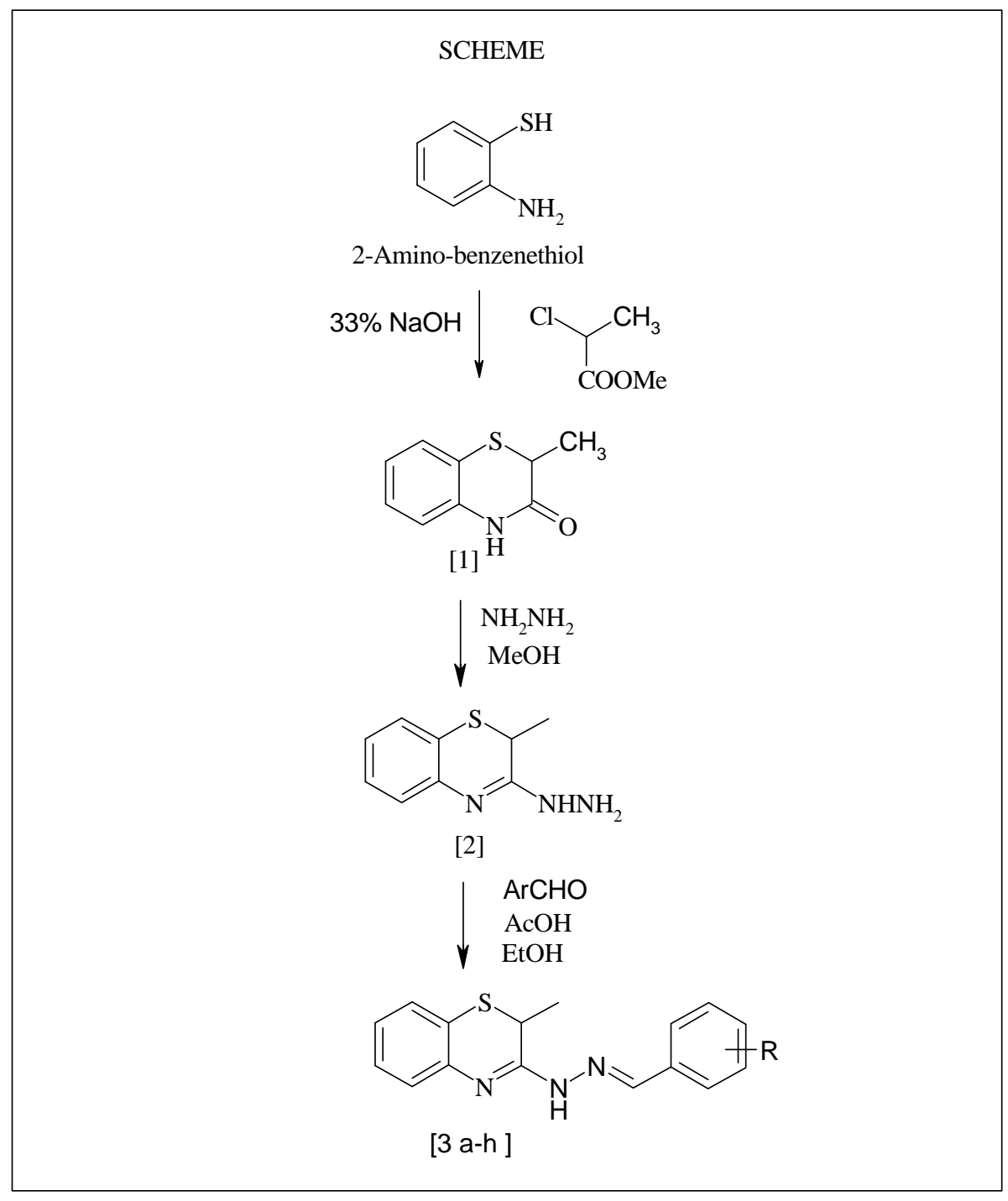

\section{Experimental}

Melting points were taken in an open capillary tube and are uncorrected. IR spectra (KBr/ Nujol) were recorded on a Perkin-Elmer 783 spectrophotometer and PMR spectra (DMSO- $\mathrm{d}_{6} / \mathrm{CDC}_{3}$ ) on a Bruker Ac 300F spectrometer $(300 \mathrm{MHz})$ using TMS as an internal standard. The mass spectra were recorded by EIMS technique on an Autospec mass spectrometer. The progress ${ }^{1-5}$ of reaction and purity of samples were checked by TLC using silica gel G. 
Synthesis of 2-methyl-1,4-benzothiazine-3(1H)-one (1):

A mixture of 2-aminothiophenol $(2 \mathrm{~g}, 0.016 \mathrm{~mol})$ and methyl 2-chloropopionate $(1.96 \mathrm{~g}, 0.016 \mathrm{~mol})$ containing $33 \% \mathrm{NaOH}(17.5 \mathrm{ml})$ was refluxed on a steam bath for $5 \mathrm{hrs}$. The reaction mixture was then cooled, neutralized with dilute $\mathrm{HC}$ and the precipitated solid was filtered, washed with water and recrystallized from $80 \%$ ethanol. Yield: $2 \mathrm{~g},(69 \%)$. M.P. $125^{\circ} \mathrm{C}$. Elemental analysis: Found: C, $61.25 ; \mathrm{H}, 4.84$; $\mathrm{N}, 6.93 \%$. for $\mathrm{C}_{9} \mathrm{H}_{9} \mathrm{ONS}$ Required $\mathrm{C}, 60.34 ; \mathrm{H}, 5.03 ; \mathrm{N}, 7.82 \%$. IR(KBr): $v_{\max } 3220(\mathrm{NH}), 1700-1650 \mathrm{~cm}^{-1}$ (cyclic $>C=0) . \quad \operatorname{PMR}\left(\mathrm{CDC}_{3}\right): \delta, 1.5\left(3 \mathrm{H}, \mathrm{d}, \mathrm{CHCH}_{3}\right), 3.5-3.6\left(1 \mathrm{H}, \mathrm{q}, \mathrm{CHCH}_{3}\right), 6.9-7.35(4 \mathrm{H}, \mathrm{m}, \mathrm{Ar}-\mathrm{H}), 9.2-9.3 \mathrm{ppm}(1 \mathrm{H}, \mathrm{s}$, exchangeable with $\mathrm{D}_{2} \mathrm{O}, \mathrm{NH}$ ).

Synthesis of 3-hydrazino-2-methyl-1,4-benzothiazine (2):

A mixture of $1(1.44 \mathrm{~g}, 0.008 \mathrm{~mol})$ and hydrazine hydrate $(0.4 \mathrm{~g}, 0.008 \mathrm{~mol})$ in methanol $(10 \mathrm{ml})$ was heated on a steam bath at $70-80^{\circ} \mathrm{C}$ for $2 \mathrm{hrs}$. The reaction mixture was concentrated and cooled to get a white crystalline product. Yield: $0.83 \mathrm{~g},(83 \%) ;$ M.P. $110^{\circ} \mathrm{C}$. Elemental Analysis: Found: C, 56.46; H, 4.87; N, 22.57\%. for $\mathrm{C}_{9} \mathrm{H}_{11} \mathrm{~N}_{3} \mathrm{~S}$, Required C, 55.95; $\mathrm{H}, 5.77 ; \mathrm{N}, 21.76 \%$. IR(KBr): $v_{\max } 3312-3100\left(\mathrm{NHNH}_{2}\right), 1665 \mathrm{~cm}^{-1}$ $(>\mathrm{C}=\mathrm{N})$.

Synthesis of 3-[arylidinehydrazino]-1H-1,4-benzothiazine (3a-h):

A mixture of $2(0.12 \mathrm{~g}, 0.006 \mathrm{~mol})$ and substituted aldehyde or ketone $(0.006 \mathrm{~mol})$ in methanol $(10 \mathrm{ml})$ was refluxed in presence of acetic acid (2-3 drops) on a steam bath for 3hrs. The reaction mixture was cooled and concentrated to get a white crystalline product. Yield: varied from (26-95\%). Additional analytical data is given in TABLE 1. 3a: IR (KBr): $v_{\max } 3200-3100(\mathrm{NH}), 1620(>C=N), 1600 \mathrm{~cm}^{-1}(\mathrm{C}=\mathrm{C}) . \quad \mathrm{PMR}$ $\left(\mathrm{CDC}_{3}\right): \delta, 1.4-1.5\left(3 \mathrm{H}, \mathrm{d}, \mathrm{CHCH}_{3}\right), 3.53-3.6\left(1 \mathrm{H}, \mathrm{q}, \mathrm{CHCH}_{3}\right), 3.94\left(3 \mathrm{H}, \mathrm{s}, \mathrm{OCH}_{3}\right), 6.63(1 \mathrm{H}, \mathrm{br} . \mathrm{s}, \mathrm{OH}$ exchangeable with $\left.\mathrm{D}_{2} \mathrm{O}\right), 6.93-7.43(7 \mathrm{H}, \mathrm{m}, \mathrm{Ar}-\mathrm{H}), 9.51\left(\mathrm{H}, \mathrm{s}\right.$, NH exchangeable with $\left.\mathrm{D}_{2} \mathrm{O}\right), 9.82(1 \mathrm{H}, \mathrm{s}, \mathrm{N}=\mathrm{CH}) .3 \mathrm{~b}$ : $\quad \mathrm{IR}(\mathrm{KBr})$ : $v_{\max }$ 3100-3200 (NH), $1620 \mathrm{~cm}^{-1}(>C=N)$ ). 3c: IR (KBr): $v_{\max } 3100-3300(\mathrm{NH}), 1615 \mathrm{~cm}^{-1}(>C=\mathrm{N}) .3 \mathrm{~d}: \quad$ IR $(\mathrm{KBr}): v_{\max } 3050-3250(\mathrm{NH}), 1615(>C=\mathrm{N}), 1050 \mathrm{~cm}^{-1}(\mathrm{C}-\mathrm{O}-\mathrm{C}) .3 \mathrm{e}: \quad \mathrm{IR}(\mathrm{KBr}): v_{\max } 3200(\mathrm{NH}), 1690-1590(>C=\mathrm{N})$, $1600 \mathrm{~cm}^{-1}(\mathrm{C}=\mathrm{C}) .3 \mathrm{ff}: \quad \mathrm{IR}(\mathrm{KBr}): v_{\max } 3500-3250(\mathrm{OH}$ or $\mathrm{NH}), 1625(>\mathrm{C}=\mathrm{N}), 1600 \mathrm{~cm}^{-1}(\mathrm{C}=\mathrm{C}) .3 \mathrm{~g}: \quad \mathrm{IR}(\mathrm{KBr}): v_{\max }$ 3550-3250 (NH), $1620(>C=N), 1600 \mathrm{~cm}^{-1}(>C=C)$. 3h: IR (KBr): $v_{\max } 3540(\mathrm{NH}), 1620(>C=N), 1600 \mathrm{~cm}^{-1}(\mathrm{C}=\mathrm{C})$.

Prediction of biological activities

The compounds $3 \mathrm{a}-3 \mathrm{~h}$ were studied for the predictions of their probabilities of being active $[\mathrm{Pa}]$ and inactive [Pi] for the selected activities such that the Pa>70\% .A software application (PASS) was used for this purpose. The relationship between structure and different biological activities was studied. It was found that 3-[arylidinehydrazino]-1H-1,4-benzothiazine (3a-h) are expected to exhibit spectacular vasodilator activity.

\section{Conclusion}

The comparison between their probabilities of being active as a potential vasodilator revealed that the compound 3-[3,4,5-trimethoxy phenylidinehydrazino]-1H-1,4-benzothiazine (3d) shows spectacular vasodilator activity. Hence this compound has been recommended for the screening of the vasodilator activity. 
TABLE 1. Physical data for compound [3a-h]

\begin{tabular}{|c|c|c|c|c|c|}
\hline \multirow{2}{*}{ Comp. } & \multirow{2}{*}{$\mathrm{R}$} & \multirow{2}{*}{$\begin{array}{c}\text { Mol. } \\
\text { formula }\end{array}$} & \multirow{2}{*}{ M.p. ${ }^{0} \mathrm{C}$} & \multicolumn{2}{|c|}{ Elemental Analysis (\%) } \\
\hline & & & & Found & Required \\
\hline $3 a$ & $\begin{array}{l}3-\mathrm{OH} \\
2-\mathrm{OCH}_{3}\end{array}$ & $\mathrm{C}_{17} \mathrm{H}_{17} \mathrm{O}_{2} \mathrm{~N}_{3} \mathrm{~S}$ & 100 & $\begin{array}{c}\mathrm{C}, 63.07 ; \mathrm{H}, \\
4.78 ; \\
\mathrm{N}, 13.15\end{array}$ & $\begin{array}{c}\text { C, 62.38; H, } \\
5.19 ; \\
\text { N, } 12.84\end{array}$ \\
\hline b & $2-\mathrm{NO}_{2}$ & $\mathrm{C}_{16} \mathrm{H}_{14} \mathrm{O}_{2} \mathrm{~N}_{4} \mathrm{~S}$ & 90 & $\begin{array}{c}\mathrm{C}, 59.28 ; \mathrm{H}, \\
4.78 ; \\
\mathrm{N}, 16.88\end{array}$ & $\begin{array}{c}\text { C, 58.89; H, } \\
4.29 ; \\
\text { N, } 17.17\end{array}$ \\
\hline C & $4-\mathrm{OH}$ & $\mathrm{C}_{16} \mathrm{H}_{15} \mathrm{ON}_{3} \mathrm{~S}$ & 122 & $\begin{array}{c}\text { C, } 65.35 ; \mathrm{H}, \\
4.86 ; \\
\text { N, } 13.10\end{array}$ & $\begin{array}{c}\text { C, 64.64; H, } \\
5.05 ; \\
\text { N, } 14.14\end{array}$ \\
\hline$d$ & $3,4,5\left(\mathrm{OCH}_{3}\right)_{3}$ & $\mathrm{C}_{19} \mathrm{H}_{21} \mathrm{O}_{3} \mathrm{~N}_{3} \mathrm{~S}$ & 104 & $\begin{array}{c}\text { C, 62.14; H, } \\
5.17 ; \\
\text { N, } 12.21\end{array}$ & $\begin{array}{c}\text { C, 61.45; } \mathrm{H}, \\
5.66 ; \\
\mathrm{N}, 11.32\end{array}$ \\
\hline e & $4-Q$ & $\mathrm{C}_{16} \mathrm{H}_{14} \mathrm{~N}_{3} \mathrm{SC}$ & 138 & $\begin{array}{c}\text { C, 61.76; H, } \\
4.12 ; \\
\text { N, } 12.62\end{array}$ & $\begin{array}{c}\text { C, 60.85; H, } \\
4.43 ; \\
\text { N, } 13.31\end{array}$ \\
\hline$f$ & $3-\mathrm{OH}, \mathrm{C}_{6} \mathrm{H}_{4}$ & $\mathrm{C}_{16} \mathrm{H}_{15} \mathrm{ON}_{3} \mathrm{~S}$ & 70 & $\begin{array}{c}\mathrm{C}, 63.83 ; \mathrm{H}, \\
4.94 ; \\
\mathrm{N}, 13.75\end{array}$ & $\begin{array}{c}\mathrm{C}, 64.64 ; \mathrm{H}, \\
5.05 ; \\
\mathrm{N}, 14.14\end{array}$ \\
\hline$g$ & $2-\mathrm{OH}, \mathrm{C}_{6} \mathrm{H}_{4}$ & $\mathrm{C}_{16} \mathrm{H}_{15} \mathrm{ON}_{3} \mathrm{~S}$ & 134 & $\begin{array}{c}\text { C, 63.82; } \mathrm{H}, \\
4.86 ; \\
\text { N, } 15.06\end{array}$ & $\begin{array}{c}\text { C, 64.64; H, } \\
5.05 ; \\
\text { N, } 14.14\end{array}$ \\
\hline $\mathrm{h}$ & 4- $-\mathrm{CH}_{3}, \mathrm{C}_{6} \mathrm{H}_{4}$ & $\mathrm{C}_{17} \mathrm{H}_{17} \mathrm{ON}_{3} \mathrm{~S}$ & 113 & $\begin{array}{c}\text { C, 64.78; } \mathrm{H}, \\
6.15 ; \\
\mathrm{N}, 12.82\end{array}$ & $\begin{array}{c}\text { C, 65.59; } \mathrm{H}, \\
5.46 ; \\
\text { N, } 13.50\end{array}$ \\
\hline
\end{tabular}




\begin{tabular}{|l|r|r|}
\hline comp & \multicolumn{1}{|l|}{$\mathrm{Pa}$} & $\mathrm{Pi}$ \\
\hline $3 \mathrm{a}$ & 0.77 & 0.01 \\
\hline $3 \mathrm{~b}$ & 0.833 & 0.007 \\
\hline $3 \mathrm{c}$ & 0.797 & 0.008 \\
\hline $3 \mathrm{~d}$ & 0.838 & 0.007 \\
\hline $3 \mathrm{e}$ & 0.802 & 0.008 \\
\hline $3 \mathrm{f}$ & 0.796 & 0.008 \\
\hline $3 \mathrm{~g}$ & 0.77 & 0.009 \\
\hline $3 \mathrm{~h}$ & 0.833 & 0.007 \\
\hline
\end{tabular}

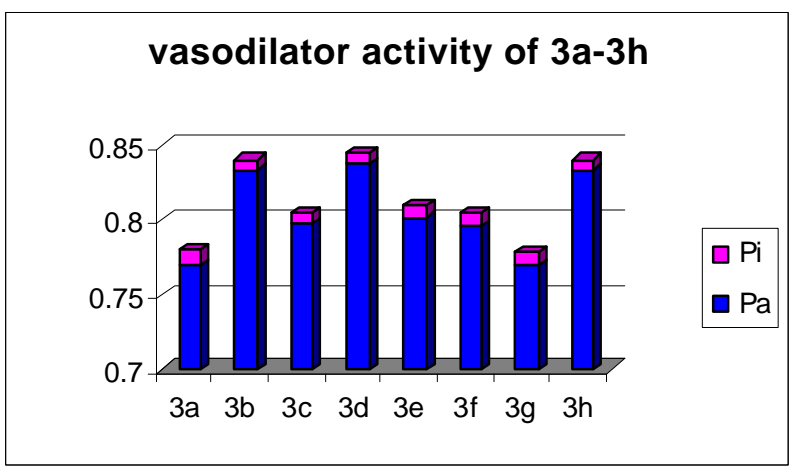

Acknowledgement

The authors thank RSIC, Punjab University, Chandigarh and IICT, Hyderabad (India) for spectral analysis.

\section{Reference}

1. Rathore R K., Gupta V, Jain M and Gupta R R, Indian J. Chem., 1993, 32B, 370.

2. Iskander G M, El Khawad I, El Shaikh, Zahran H B and Schlemper E O, J. Pakt. Chem. 1989, 331, 82 ; Chem. Abstr., 1990, 112, 357794.

3. Sastry C, Rao K, Krishnan V, Rastogi K, Lovekar C, Tripathi H, Kondaiah S and Sai G, Ind. J. Chem., 1990, 29B(3), 297.

4. Hogale $M$ and Uthale A, Proc. Ind. Acad. Sci.(Chem. Sci.), 1990, 102(4), 535; Hogale M. and Uthale A , 1990, Ind. J. Chem., 29B, 590.

5. Nacci V, Campiani G and Garofelo A, J. Heterocyclic Chem. , 1990, 27, 1329, 1990. 


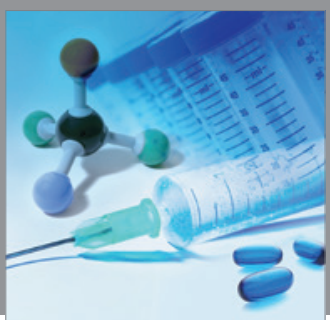

International Journal of

Medicinal Chemistry

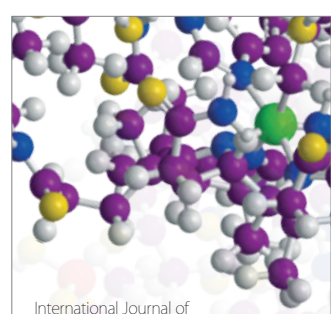

Carbohydrate Chemistry

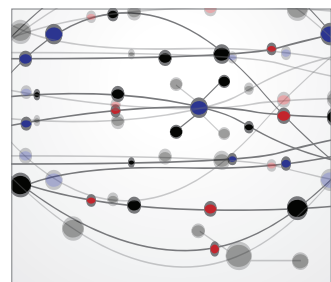

The Scientific World Journal
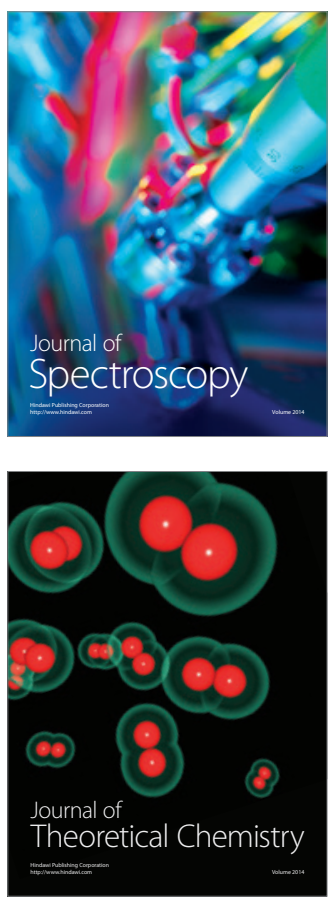
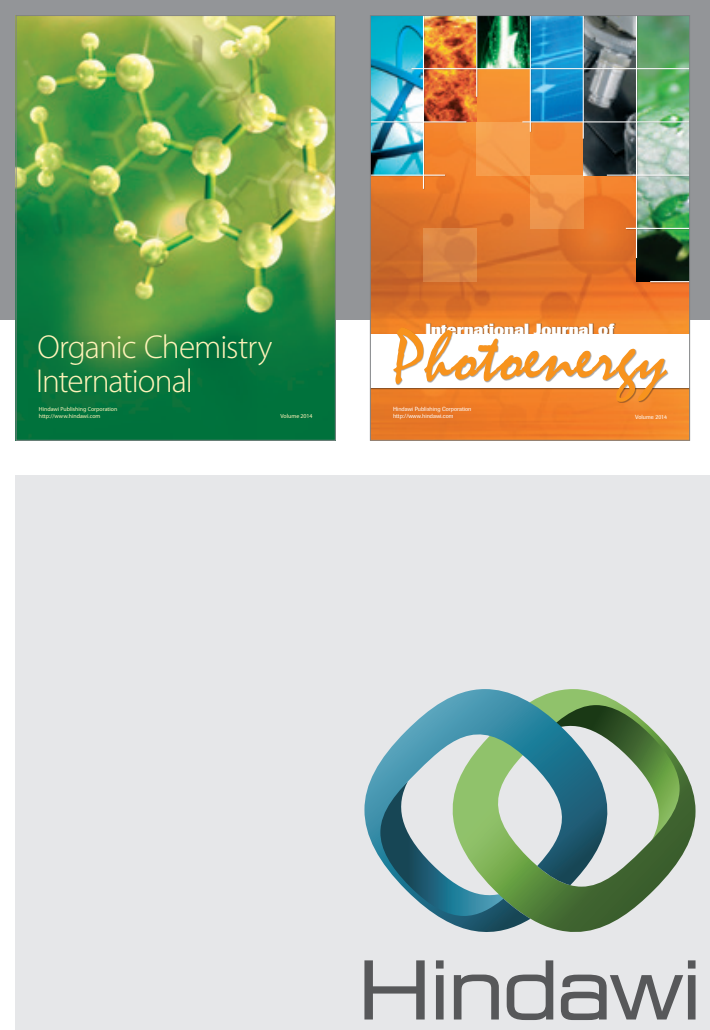

Submit your manuscripts at

http://www.hindawi.com
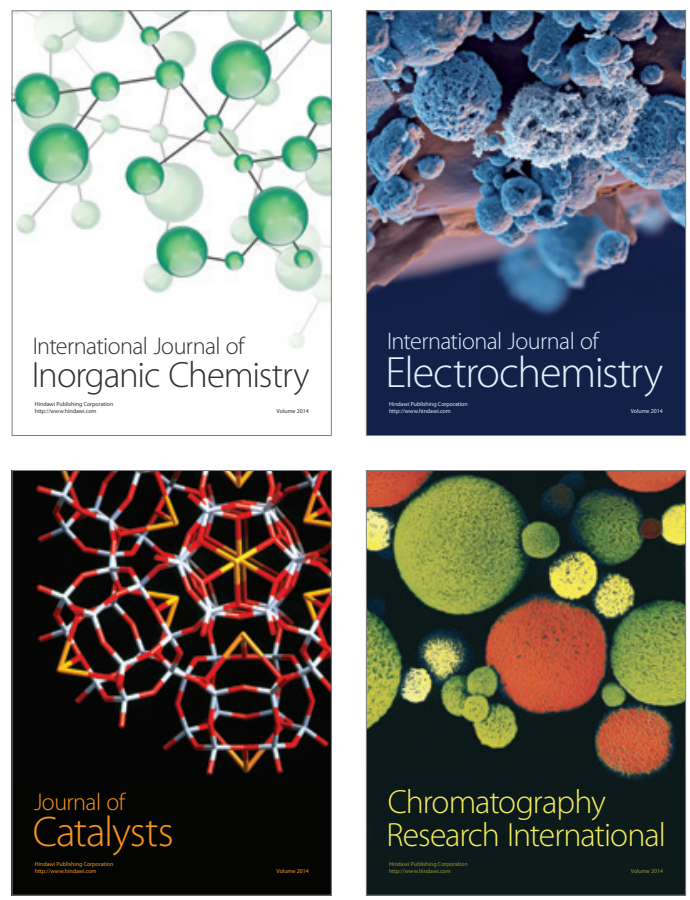
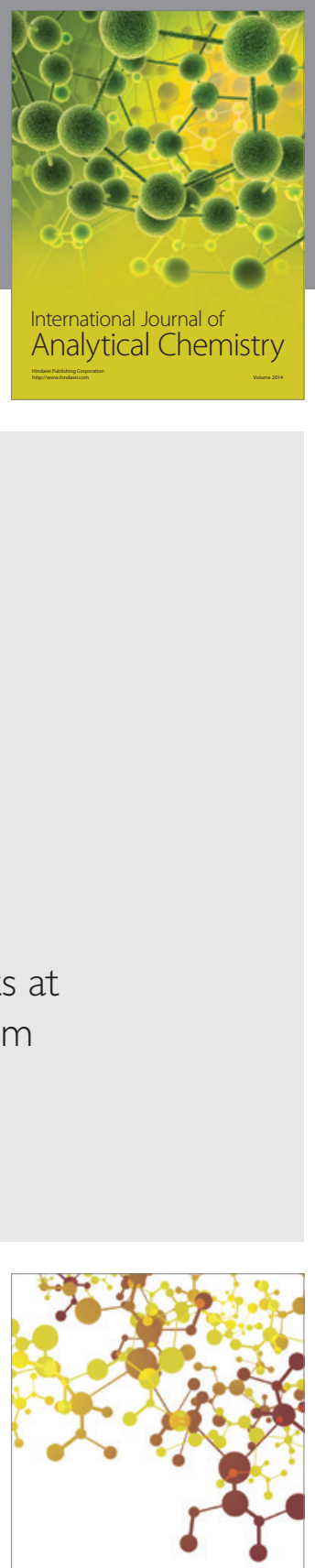

Journal of

Applied Chemistry
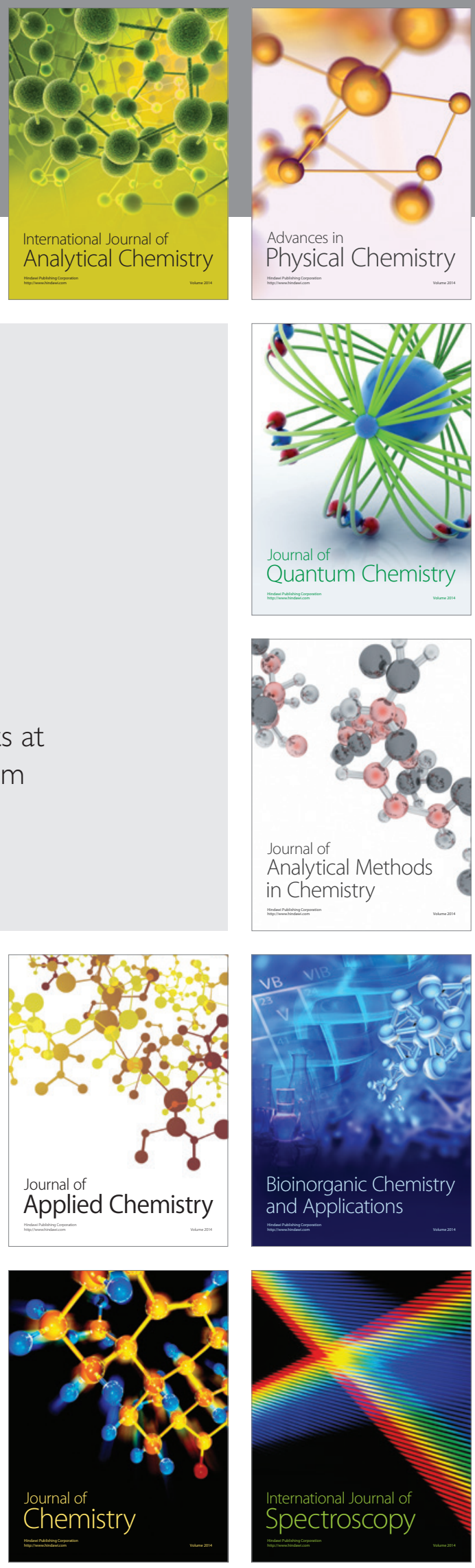